\title{
Scales and scaling in past climate data
}

\author{
Christian Franzke \\ 1st CVAS workshop, Hamburg, Germany, 28-30 November 2016
}

The aim of the Climate Variability Across Scales (CVAS) working group is to quantify climate variability across space and time on centennial and millennial time scales which is relevant for better constraining future climate evolution. A second interesting aspect of climate variability on these time scales is that it is strong but cannot be explained by changes in orbital forcings. This suggests that internal, nonlinear, climatic processes dominate. Better understanding of these processes would allow a more accurate evaluation and to better constrain climate models. However, the description and understanding of centennial and millennial climate variability requires long-term climate datasets. Such datasets need to be based on proxy data and be developed by paleoclimatologists, climatologists and climate modelers, combined with nonlinear multi-scale and scaling analysis techniques developed by nonlinear geophysicists and statisticians.

In this workshop, we brought together leading scientists from these two community groups. In order to facilitate knowledge exchange, we had 11 tutorial-style, introductory talks together with shorter contributory talks and posters. About half of the introductory talks covered the themes of the physical processes leading to scaling, stochastic dynamical systems and linear response, and the spatial pattern of variability. Scaling is a geometric concept by which an object is enlarged or shrunk by a linear transformation and is described by a power law. The other half of the workshop focused more on scaling in proxy data. We also had presentations on the potential pitfalls and issues with proxy data sets and recording systems in

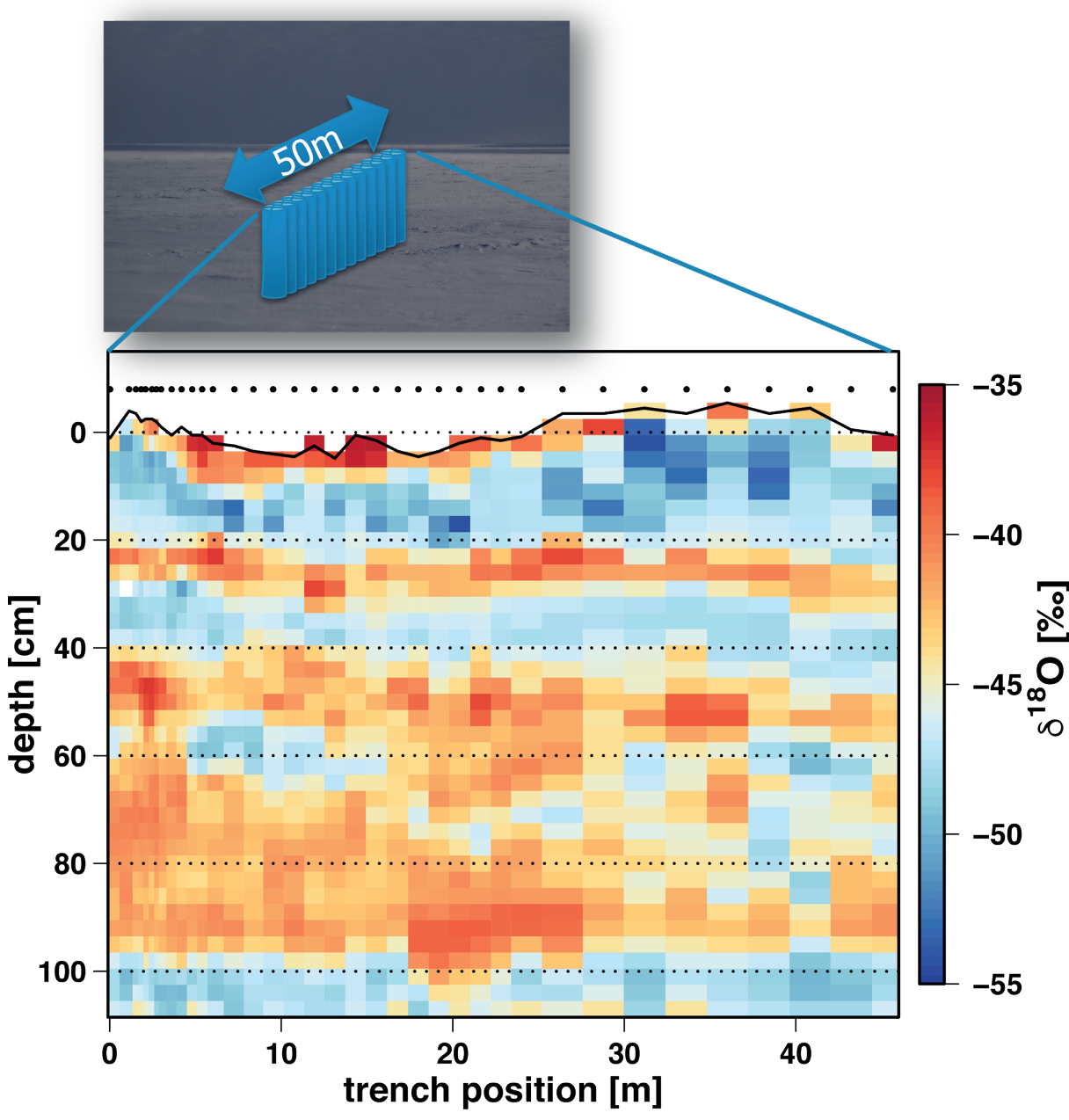

Figure 1: A two-dimensional view (horizontal $x$ vertical) of oxygen isotopes $\left(\delta^{18} \mathrm{O}\right)$ in Antarctic snow near the EPICA deep ice-core drilling site (modified from Münch et al. 2016). Vertically alternating layers of enriched and depleted isotopic composition represent the climatic seasonal cycle. In addition to that, strong horizontal variability - stratigraphic noise - is visible. Such studies investigating the spatial structure of proxy data allow to quantify and correct for non-climatic variability in climate proxy records. general, and discussed these issues for ice cores in particular. Thom Laepple showed some intriguing results from recent measurements using snow trenches (Fig. 1) and how the proxy data vary across rather short distances. This suggests that the recording systems are intrinsically noisy and we should examine and perhaps even combine multiple measurements if possible. This highlights the need to be careful when interpreting the variability in proxy data, especially in a global context.

Part of the workshop was dedicated to discussions about the future directions of the working group. One identified outcome is the need for a data repository useful for centennial to millennial scale variability, together with case studies which describe the potential pitfalls of the respective data set and analysis tools, and readily available software allowing scientists to obtain reproducible results. Such a repository should also provide information such as the space-time resolutions of the data and all uncertainties.

As important research questions, we identified:

-What is the spatial pattern of centennial to millennial scale variability, especially in the Holocene? Do existing climate models reproduce these spatial patterns?

-What are the scaling regimes in the Holocene? Are there differences between the tropics, extratropics and the polar regions?

- Can we systematically separate or quantify internal from externally forced variability?

The CVAS working group had a session and conducted a short course at the EGU meeting in Vienna in April this year. The next workshop will take place in Potsdam 25-27 October 2017. The group also agreed on organizing a Past Global Changes Magazine special issue on scaling of climate variability.

The 1st CVAS workshop was supported by the German Research Foundation (DFG), through the cluster of excellence CliSAP (EXC177) at the University of Hamburg, and PAGES.

\section{AFFILIATIONS}

Meteorological Institute and Center for Earth System Research and Sustainability, University of Hamburg, Germany

CONTACT

Christian Franzke: christian.franzke@uni-hamburg.de REFERENCES

Münch T et al. (2016) Clim Past 12: 1565-1581 